\title{
Análise Crítica do Uso dos Índices do Homeostasis Model Assessment (HOMA) na Avaliação da Resistência à Insulina e Capacidade Funcional das Células- $\beta$ Pancreáticas
}

revisão

\author{
ana Carolina J. Vasques \\ LINA ENRIQUETA F. P. L. ROSADO \\ Rita de Cássia G. Alfenas \\ Bruno Geloneze
}

Departamento de Nutrição e

Saúde da Universidade Federal

de Viçosa MG, Brasil (ACJV,

LEFPLR, RCGA); Laboratório de

Investigação em Metabolismo e

Diabetes - Endocrinologia -

Faculdade de Ciências Médicas

da Universidade Estadual de

Campinas (Unicamp), Campinas, SP, Brasil (BG).

Recebido em 01/06/2007

Aceito em 31/08/2007
RESUMO

A disfunção das células- $\beta$ e a resistência insulínica são anormalidades metabólicas inter-relacionadas na etiologia do diabetes tipo 2. Em diversos países, tem sido observado o aumento da prevalência de obesidade e diabetes em associação com a presença da resistência insulínica. Nesse contexto, é útil a mensuração da resistência insulínica e da capacidade funcional das células- $\beta$ nos indivíduos. Os índices Homeostasis Model Assessment (HOMA) têm sido amplamente utilizados, representando uma das alternativas para avaliação desses parâmetros, principalmente por figurarem um método rápido, de fácil aplicação e de menor custo. Esta revisão discute sobre a origem e a evolução dos índices HOMA, bem como as particularidades do método, abordando aspectos relacionados à sua validação e aos pontos de corte existentes para sua interpretação. (Arq Bras Endocrinol Metab 2008;52/1:32-39)

Descritores: Resistência à insulina; Índice HOMA; Glicemia; Insulinemia

\section{ABSTRACT}

Critical Analysis on the Use of the Homeostasis Hodel Assessment (HOMA) Indexes in the Evaluation of the Insulin Resistance and the Pancreatic Beta Cells Functional Capacity.

Beta-cell dysfunction and insulin resistance are interrelated metabolic abnormalities in the aetiology of Type 2 Diabetes. In several countries, increases in the prevalence of obesity and diabetes have been observed in association with the presence of insulin resistance. In this context, measurement of insulin resistance and beta-cell function is useful. The HOMA indexes (Homeostasis Model Assessment) have been widely used, representing an alternative for the evaluation of these parameters, particularly as a fast, easy and cheap method. This review discusses the origin and evolution of the HOMA index, as well as details of the method, analyzing features related to its validation and the cutoff limits for its interpretation. (Arq Bras Endocrinol Metab 2008;52/1:32-39)

Keywords: Insulin resistance; HOMA index; Blood glucose; Insulinemia

\section{INTRODUÇÃO}

MANUTENÇÃO DA GLICEMIA NORMAL DEPENDE principalmente da capacidade funcional das células- $\beta$ pancreáticas $(\mathrm{BcC})$ em secretar insulina e da sensibilidade tecidual à ação da insulina $(S I)(1)$. A disfunção das células- $\beta$ e a resistência insulínica (RI) são anormalidades metabólicas inter-relacionadas na etiologia do diabetes melito do tipo 2 (DM2) (1,2). A RI caracteriza-se por falhas das células-alvo em responder aos níveis normais de insulina circulantes, 
resultando hiperinsulinemia compensatória na tentativa de se obter uma resposta fisiológica adequada $(3,4)$.

Estudos têm identificado a RI como um fator preditor independente de DM2 (5-7). Em virtude de associação entre RI e disfunção endotelial, passo inicial para o processo de aterosclerose $(8,9)$, também tem-se considerado como fator preditor independente de doença cardiovascular (10-12). Em vários países, a incidência crescente de RI, associada ao aumento das prevalências de obesidade e DM2, tem sido observada (13-18).

Dessa forma, torna-se necessária a utilização de técnicas apropriadas para mensuração da $\mathrm{RI}$ e da $\mathrm{BcC}$ nos indivíduos. $\mathrm{O}$ teste de tolerância endovenosa à glicose com amostras freqüentes - Frequent Sample IV Glucose Tolerance Test (FSIVGTT) (19) para RI e as técnicas de clamp euglicêmico e clamp hiperglicêmico para RI e da $\mathrm{BcC}$, respectivamente, proporcionam uma mensuração direta desses parâmetros, uma vez que analisam os efeitos de uma quantidade pré-determinada de insulina exógena administrada no indivíduo, além de mensurar a quantidade de insulina metabolizada pelos tecidos periféricos durante a estimulação com insulina (20).

Embora atualmente o clampe euglicêmico-hiperinsulinêmico seja considerado a técnica padrão-ouro disponível, ou seja, de maior acurácia para avaliação da RI in vivo, é dispendioso, demorado, invasivo e de alta complexidade, sendo inviável sua aplicação em estudos populacionais e na prática clínica $(1,21)$.

Durante as últimas décadas, métodos alternativos para a avaliação da RI e da BcC têm sido propostos $(19,22)$. O Modelo de Avaliação da Homeostase, mais conhecido como índice HOMA, representa uma das alternativas à técnica de clampe para avaliação da $\mathrm{RI}$ e da $\mathrm{BcC}$, fornecendo uma medida indireta da $\mathrm{RI}$ ao avaliar, em condições de homeostase e jejum, a insulina endógena e a glicemia (22). Esse método vem sendo amplamente utilizado, principalmente em estudos envolvendo um grande número de participantes, por ser um método de fácil aplicação, rápido e de menor custo (11,16,23-25).

Diante da importância desse método, o presente estudo objetivou revisar a literatura no intuito de descrever a origem e a evolução dos índices HOMA, bem como as particularidades do método, sua validação e os pontos de corte existentes para tais índices.

\section{ÍNDICES HOMA}

\section{Histórico e bases fisiológicas}

Em 1976, Turner e cols. (26) postularam que os níveis insulinêmicos e glicêmicos de jejum eram predominan- temente regulados por uma relação de feedback entre o fígado e as células- $\beta$. Segundo essa proposta, uma redução na secreção de insulina levaria o fígado a aumentar a glicemia basal até que os níveis normais de insulina fossem estabelecidos, inibindo, posteriormente, a secreção de glicose pelo fígado por um feedback negativo. Desse modo, a glicemia basal seria uma função da $\mathrm{BcC}$, e a hiperglicemia seria mantida por uma redução na insulinemia. Por outro lado, o aumento da RI necessitaria de um acréscimo nos níveis de insulina na veia porta com o objetivo de limitar a liberação de glicose, e a glicemia basal seria ligeiramente aumentada até que os níveis suficientes de insulina fossem produzidos. Assim, a insulinemia basal representaria uma função da RI. Se um indivíduo com capacidade funcional da célula- $\beta$ reduzida se tornasse obeso, desenvolvendo RI, a glicemia basal tenderia a ser ainda mais elevada com o objetivo de manter níveis insulínicos aumentados na tentativa de vencer a RI (Figura 1).

A partir desse modelo teórico, postulou-se que a deficiência da secreção insulínica pelas células- $\beta$ poderia ser determinada a partir da hiperglicemia apresentada, uma vez conhecida a quantidade de insulina secretada para determinada concentração glicêmica. Partindo dos resultados obtidos em estudos experimentais com animais e humanos, foi construído um modelo matemático para estimar o nível de funcionamento das células- $\beta$ e a SI, os quais seriam proporcionais aos níveis glicêmicos e insulinêmicos no estado basal ou steady state (27). A partir desse modelo, seria possível avaliar a contribuição da disfunção da célula- $\beta$ e da RI na etiologia do DM, possibilitando a seleção do tratamento mais apropriado para tal doença (28). Entretanto, o modelo citado não fazia distinção entre a SI hepática e a periférica, assumindo que a RI afeta de forma idêntica o fígado e os tecidos periféricos.

No ano de 1985, Matthews e cols. publicaram um modelo mais abrangente chamado Homeostasis Model Assessment (HOMA) - Modelo de Avaliação da Homeostase. Esse modelo matemático prediz o nível de RI e $\mathrm{BcC}$ de acordo com a glicemia e a insulinemia basal. Dessa forma, pelo gráfico do HOMA, os valores de glicemia e insulinemia basal podem ser traçados para predizer o nível de $\mathrm{RI}$ e $\mathrm{BcC}(22)$.

$\mathrm{O}$ modelo citado anteriormente foi ajustado, dando origem a duas equações simplificadas: $\mathrm{HOMAl-IR}\left(\mathrm{Ho}^{-}\right.$ meostasis Model Assessment Insulin Resistance $)=(\mathrm{IJ} \times \mathrm{GJ}) /$ 22,5 e HOMAl-\%B (Homeostasis Model Assessment $\beta$-Cell Function $)=(20 \times \mathrm{IJ}) /(\mathrm{GJ}-3,5)$, as quais podem ser usadas para estimar a $\mathrm{RI}$ e a $\mathrm{BcC}$, respectivamente. Em tais 


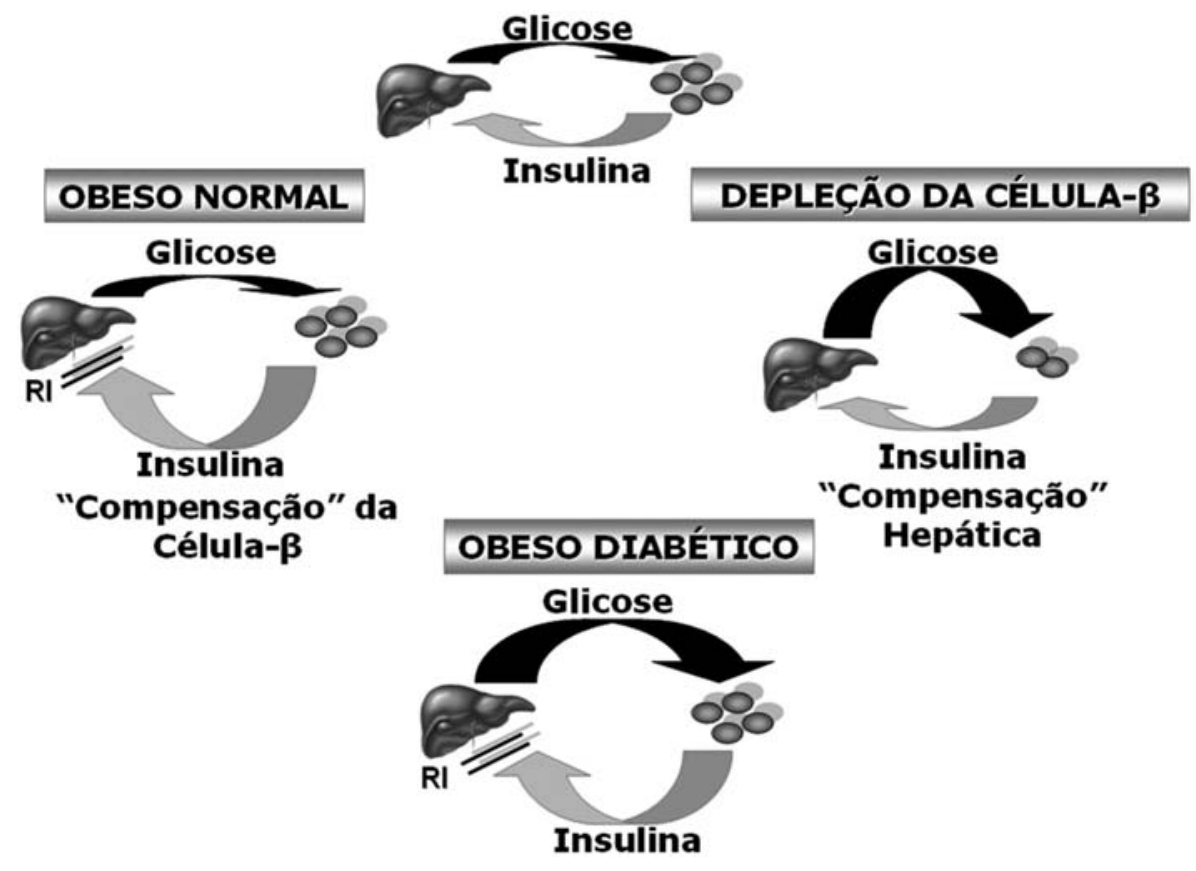

Figura 1. Relação de feedback entre o fígado e as células- $\beta$ pancreáticas em indivíduos saudáveis, em obesos com Rl, em indivíduos com disfunção da célula- $\beta$ e em obesos diabéticos (26).

equações, IJ corresponde à insulinemia de jejum em $\mathrm{mU} / \mathrm{L}, \mathrm{e} \mathrm{GJ}$, à glicemia de jejum em mmol/L (22).

Em substituição às dosagens de insulina total de jejum, determinada por radioimunoensaio, também são utilizados ensaios específicos para insulina ou o peptídeo C (22). Entretanto, como a determinação do nível de peptídeo C é uma medida robusta da secreção insulínica, não sendo considerado um indicador da ação insulínica, sua determinação deve ser utilizada apenas para o cálculo do HOMA-B\%. Na prática, tem sido utilizada apenas a insulinemia para cálculo de ambos os índices, uma vez que a vantagem teórica citada anteriormente não compensa os gastos e o trabalho adicional para análise das amostras (29).

Mais recentemente, foi publicada uma atualização do modelo original, o HOMA2, com bases fisiológicas mais precisas na predição da resposta homeostática. En- tre tais modificações, estão a distinção entre RI hepática e periférica, a incorporação da estimativa de secreção de pró-insulina ao modelo, viabilizando a utilização de ensaios específicos ou não para insulina e, por último, a modificação na curva de secreção insulínica e a inclusão ao modelo da perda renal de glicose, possibilitando a avaliação da $\mathrm{RI}$ e da $\mathrm{BcC}$ em concentrações glicêmicas $>10 \mathrm{mmol} / \mathrm{L}(30)$.

Além de tais modificações, também foi desenvolvido o programa de computador HOMA Calculator, com o objetivo de viabilizar e tornar mais rápidos os cálculos da SI $(\% \mathrm{~S})$ e da $\mathrm{BcC}(\% \mathrm{~B})$ (30). Esse programa aceita apenas valores correspondentes a um estado de homeostase, ou seja, valores glicêmicos muito baixos não seriam adequados, pois estariam relacionados a um estado de hipoglicemia (29) (Tabela 1).

Tabela 1. Faixas das dosagens viáveis para cálculo dos índices HOMA2-\%S e HOMA2-\%B no programa HOMA Calculator (31).

\begin{tabular}{ll}
\hline Parâmetro bioquímico & Dosagem \\
\hline Glicemia de jejum & $3,5-25,0 \mathrm{mmol} / \mathrm{L}$ ou $63-450 \mathrm{mg} / \mathrm{dL}$ \\
\hline Insulinemia de jejum & $20-400 \mathrm{pmol} / \mathrm{L}$ \\
Insulina plasmática específica & $20-300 \mathrm{pmol} / \mathrm{L}$ \\
Peptídeo C plasmático & $0,2-3,5 \mathrm{nmol} / \mathrm{L}$ \\
\hline
\end{tabular}




\section{Considerações técnicas sobre a utilização dos índices HOMA}

A mensuração da insulinemia deve ser feita em condições precisas e reprodutíveis para a determinação da RI e da BcC pelo HOMA. O grau de especificidade analítica do ensaio utilizado para medir a insulinemia depende dos níveis de reação cruzada com a pró-insulina. Indivíduos com maior grau de RI apresentam maiores concentrações de pró-insulina. Assim, um ensaio pouco sensível para a insulina, apresentando elevada taxa de reação cruzada com pró-insulina, poderá resultar em falsos-positivos para hiperinsulinemia. A utilização de métodos de radioimunoensaio com duplo-anticorpo é recomendada, já que permitem pouca ou nenhuma reação cruzada com a pró-insulina e seus derivados (32).

É fundamental ainda destacar que a hemólise das amostras sanguíneas deve ser evitada, uma vez que, quando ela ocorre, há liberação de conteúdo eritrocitário para o soro, resultando liberação de enzimas proteolíticas, que podem degradar hormônios peptídicos (33). Estudo realizado in vitro identificou degradação da insulina em até $25 \%$, podendo levar à subestimação das dosagens (34).

Outro aspecto importante a ser considerado é o número de amostras de sangue coletadas. Ao avaliar a reprodutibilidade do índice HOMA em 18 homens diabéticos ou com tolerância normal à glicose (TNG), foi verificado um coeficiente de variação de $32 \%$ (22). Mais recentemente, em estudo realizado com 30 indivíduos, identificou-se que a utilização de uma amostra única de sangue apresenta um coeficiente de variação intra-individual de 10,3\% para HOMA-IR e de 7,7\% para HOMA-\%B, comparados com coeficiente de variação de $5,8 \%$ e 4,4\%, respectivamente, quando é utilizada a média de três amostras (35).

Essas variações indicam que a coleta de uma única amostra sanguínea não seria confiável para estimativa clínica da RI e da BcC pelo HOMA. Como a liberação de insulina oscila, já que é liberada em ritmo pulsátil, devem ser coletadas, pelo menos, três amostras de sangue em jejum, uma a cada cinco minutos por 15 minutos. Esse é o tempo necessário para que um ciclo de secreção desse hormônio seja completado (22).

No entanto, no mesmo estudo citado anteriormente, houve correlação muito forte e altamente significante $(\mathrm{r}=0,99 ; \mathrm{p}<0,0001)$ entre a insulinemia obtida de uma única amostra com a insulinemia média de três amostras de sangue, tomadas em intervalos de cinco minutos, inferindo que, em estudos populacionais, a utilização de apenas uma amostra sanguínea seria suficiente para cálculo dos índices HOMA (35). Em concordância, outros trabalhos encontraram resultados semelhantes em indivíduos diabéticos e não-diabéticos $(32,36)$.

A interpretação dos resultados obtidos utilizando o HOMA também deve ser realizada com cautela. A avaliação da $\mathrm{BcC}$ não deve ser realizada de forma isolada e sim em conjunto com a avaliação da RI. Existem indivíduos que possuem elevada SI e, por isso, secretam menor quantidade de insulina. Nesses casos, a avaliação isolada da $\mathrm{BcC}$ poderia levar ao diagnóstico falso-positivo para defeito na secreção insulínica (29).

\section{Validação dos índices HOMA}

A utilidade de medidas alternativas para a estimativa da $\mathrm{RI}$ e da $\mathrm{BcC}$ depende do grau em que se correlacionam com as medidas diretas de avaliação desses parâmetros, como as técnicas de clamp (20) ou o teste de tolerância endovenosa à glicose com amostras freqüentes - FSIVGTT (19).

O primeiro trabalho de validação do HOMAl envolveu uma amostra de homens com TNG ou portadores de DM2. As correlações encontradas para o HOMAl-IR $(\mathrm{r}=0,88 ; \mathrm{p}<0,000 \mathrm{l})$ e HOMAl-B $\%(\mathrm{r}=0,6 \mathrm{l} ; \mathrm{p}<0,0 \mathrm{l})$ em relação à técnica de clampe euglicêmico foram fortes e altamente significantes. Entretanto, o estudo incluía apenas indivíduos do sexo masculino, além do número de participantes ser pequeno $(\mathrm{n}=22)$ para um estudo de validação (22).

Bonora e cols., avaliando 115 italianos (66 homens e 49 mulheres), de 19 a 67 anos com variados graus de tolerância à glicose e SI, também identificaram correlação forte e altamente significante entre o HOMAl-IR e o clampe euglicêmico $(\mathrm{r}=-0,85 ; \mathrm{p}<$ $0,0001)$, além da concordância entre as duas técnicas ter sido boa (kappa $=0,63)$. Vale ressaltar que, nesse estudo, o tamanho da amostra foi maior que o apresentado anteriormente e que, ao fazer distinções entre sexo, idade, condição de hipertenso ou normotenso, presença ou ausência de obesidade e DM2 ou tolerância normal à glicose, os resultados de correlação foram consistentes para todas essas categorias (32).

No estudo de Lansang e cols., foram avaliados 63 americanos (49 homens e 14 mulheres) de 18 a 60 anos, incluindo indivíduos eutróficos e obesos. O HOMAl-IR foi considerado uma alternativa à técnica de clampe euglicêmico para a avaliação da RI em hiperten- 
$\operatorname{sos}(\mathrm{r}=-0,64 ; \mathrm{p}<0,0001)$ e normotensos $(\mathrm{r}=-0,58$; $\mathrm{p}<0,03)$ não-diabéticos $(37)$.

Um ensaio clínico foi conduzido durante seis semanas, envolvendo 55 japoneses ( 44 homens e 11 mulhe-

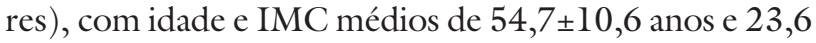
$\mathrm{kg} / \mathrm{m}^{2}$, respectivamente. Após intervenção com dieta, exercício físico e tratamento com hipoglicemiante oral, o HOMAl-IR mostrou-se útil não só para diagnóstico $(\mathrm{r}=-0,613 ; \mathrm{p}<0,0001)$, mas também para o acompanhamento da evolução dos pacientes diabéticos $(\mathrm{r}=$ $-0,734 ; \mathrm{p}<0,0001)$, uma vez que, assim como o clampe euglicêmico, conseguiu identificar melhoras na RI após o tratamento (36).

Em estudo envolvendo a participação de 156 crianças e adolescentes americanos ( 64 do sexo masculino e 92 do sexo feminino), incluindo eutróficos e com excesso, as correlações encontradas entre o HOMAl-IR e o clampe euglicêmico foram muito fortes e altamente significantes $(\mathrm{r}$ $=0,91 ; \mathrm{p}<0,01)$. Naquele mesmo estudo, o HOMAl-B $\%$ também correlacionou-se fortemente com o clampe hiperglicêmico, tanto na avaliação da primeira $(r=0,82$; $\mathrm{p}<0,01)$ quanto na segunda fase de secreção insulínica $(\mathrm{r}=0,86 ; \mathrm{p}<0,01)$, mostrando-se um método valioso para estimativa da $\mathrm{RI}$ e da $\mathrm{BcC}$ (38).

Por outro lado, um estudo realizado com 90 coreanos (76 homens e 14 mulheres), adultos e idosos e com variados graus de tolerância à glicose, identificou limitações no HOMAl-IR em relação ao clampe euglicêmico para predizer a $\mathrm{RI}$ em indivíduos com menor IMC $(\mathrm{r}=-0,441 ; \mathrm{p}<0,001)$, menor $\mathrm{BcC}(\mathrm{r}=-0,527$; $\mathrm{p}<0,001)$ e maiores níveis glicêmicos $(\mathrm{r}=-0,52 ; \mathrm{p}<$ $0,001)$, uma vez que as correlações foram moderadas para esses grupos (34).

Dois outros estudos validaram o HOMAl em relação ao FSIVGTT. O primeiro avaliou 162 caucasianos (86 homens e 76 mulheres), com TNG e idade entre 30 a 65 anos, sendo observada correlação moderada e altamente significante entre os métodos $(\mathrm{r}=-0,53 ; \mathrm{p}<$ 0,001) (39). Já no estudo de Chang e cols., realizado com 214 norte-americanos, de 19 a 88 anos, o HOMAl-IR e o FSIVGTT identificaram padrões semelhantes de RI crescente nos indivíduos mais velhos comparados aos mais jovens $(\mathrm{r}=-0,68$; $\mathrm{p}<0,0001)$, e a concordância entre ambas as técnicas foi moderada $($ kappa $=0,51)$. Contudo, a correlação $(r=0,37, p<$ $0,0001)$ em toda a amostra e a concordância (kappa = $0,35)$ nos idosos com TDG entre o HOMA-B\% e a $\mathrm{BcC}$ avaliada pelo FSIVGTT foram fracas. Nesse mesmo estudo, o HOMA2-\%S e o HOMA2-B\% também foram comparados ao FSIVGTT e apresentaram comportamento semelhante ao HOMAl-IR e HOMAl-B\%, respectivamente $(40)$.

\section{Pontos de corte para os índices HOMA}

Entre os principais fatores que influenciam a sensibilidade insulínica, destacam-se: a deposição de gordura visceral (41), a perda de massa muscular, o envelhecimento (42), o sedentarismo (43), as diferenças étnicas $(23,44,45)$ e os fatores dietéticos (46). Em virtude de tais fatores, um dos aspectos importantes a ser observado na aplicação do HOMA com sucesso em uma dada população é a presença de pontos de corte específicos para a raça ou a faixa etária apresentada pela população estudada ou pontos de corte para populações que se assemelhem ao máximo àquela em estudo. Para a comparação das prevalências de RI entre diferentes populações, é necessário, primeiramente, o estabelecimento de valores "normais" de HOMA para cada população (29). O diagnóstico da resistência à insulina é de relevância na avaliação da presença de síndrome metabólica, que se destaca como risco para o desenvolvimento de diabetes melito tipo 2 e de doença cardiovascular aterosclerótica.

Embora o índice HOMA venha sendo amplamente utilizado, há pouco consenso quanto aos pontos de corte para a classificação da RI, o que dificulta sua utilização na pesquisa e, principalmente, na prática clínica. Além do mais, ainda não existe padronização entre os laboratórios quanto aos tipos de ensaios utilizados para a determinação da insulina plasmática, o que também é um obstáculo para sua aplicação de forma individual para diagnósticos em virtude da grande variabilidade encontrada nos resultados entre diferentes laboratórios (47).

Em sua publicação original, o modelo HOMA foi ajustado de forma que um indivíduo saudável, com idade inferior a 35 anos, apresentasse um valor de HOMAl-IR igual a 1 e uma HOMAl-\%B igual a $100 \%$ (22). Na tabela 2 , encontram-se os pontos de corte para o HOMAl-IR determinados em diversas populações e faixas etárias. Alguns desses trabalhos utilizaram-se da construção de curvas Receiver Operating Characteristic (ROC) $(24,48,49)$ para a determinação de valores ótimos para pontos de corte com base na sensibilidade e especificidade do teste. Outros autores utilizaram valores com base em médias (50-53), quintis $(55)$ ou percentis $(55,56)$ obtidos a partir de estudos populacionais. Estudos realizados com o HOMA2-IR ainda são escassos e não foram encontrados pontos de corte relacionados a esse modelo de avaliação. 
Tabela 2. Pontos de corte propostos para o HOMA1-IR.

\begin{tabular}{|c|c|c|}
\hline Ref. & Características da amostra & Valor de HOMA I-IR \\
\hline 24 & 976 coreanos de 30-79 anos, não-diabéticos & $\geq 2,34$ (curva ROC) \\
\hline 48 & 140 espanhóis, 7-16 anos, eutróficos e obesos & Próximo de 3,0 (curva ROC) \\
\hline 49 & 57 turcos, crianças e adolescentes obesos com RI e sem RI & $>3,16$ (curva ROC) \\
\hline 50 & 490 espanhóis, 19-70 anos, IMC: 26,3 × 4,4 (18-42kg/m²) e não-diabéticos & $2,7 \pm 0,1$ \\
\hline 51 & 120 chilenos, 19-40 anos, eutróficos e TNG & $1,96 \pm 0,57$ \\
\hline 52 & 2.264 brasileiros, 4-93 anos & $\begin{array}{l}\text { Idade }<18 \text { anos: } 2,39 \pm 1,93 \\
\text { Idade } \geq 18 \text { anos: } 3,20 \pm 3,56\end{array}$ \\
\hline 53 & 1.898 brasileiros de 18-90 anos com glicemia de jejum < 99mg/dL. & $\begin{array}{l}\text { Total: } 1,8 \pm 0,9 \\
\mathrm{IMC}<25 \mathrm{~kg} / \mathrm{m}^{2}=1,2 \pm 0,65 \\
\mathrm{IMC} \text { de } 25 \text { a } 30 \mathrm{~kg} / \mathrm{m}^{2}=1,8 \pm 0,98 \\
\mathrm{IMC}>30 \mathrm{~kg} / \mathrm{m}^{2}=2.9 \pm 1.6\end{array}$ \\
\hline 54 & 225 italianos, 40-79 anos, eutróficos e TNG & $>$ 2,77 (último quintil) \\
\hline 55 & 97 espanhóis, 20-65 anos, IMC = 22,2 $\pm 1,9$ kg/m², sem RI & $\begin{array}{l}\text { Total: } \geq 3,8 \text { (p 90) } \\
\text { Homens: } \geq 3,5 \text { (p 90) } \\
\text { Mulheres: } \geq 3,9 \text { (p 90) }\end{array}$ \\
\hline 56 & $\begin{array}{l}\text { 1.317 brasileiros, } 40 \pm 12 \text { anos, } \mathrm{IMC}=34 \pm 10 \mathrm{~kg} / \mathrm{m}^{2} \text { e sem alterações } \\
\text { metabólicas }\end{array}$ & $>2,71(\mathrm{p} \mathrm{90)}$ \\
\hline
\end{tabular}

IMC = índice de massa corporal, $\mathrm{P}=$ percentil, RI = resistência insulínica, ROC = Receiver Operating Characteristic, TNG = tolerância normal à glicose.

\section{CONCLUSÃO}

Desde sua publicação, o modelo HOMA passou por alterações que têm tornado seus resultados cada vez mais precisos e de mais fácil obtenção. Embora essa não seja a técnica com maior acurácia existente, ela representa uma ferramenta útil para estudos populacionais, em virtude da facilidade de sua aplicação e da correlação forte e significante com as técnicas diretas de avaliação da RI e da BcC observadas na maioria dos trabalhos de validação. Para diagnóstico ou acompanhamento individual, sua utilização ainda requer cautela nas questões relacionadas à amostragem sanguínea e à padronização do ensaio a ser utilizado pelos laboratórios na dosagem de insulina. Apesar de os estudos ainda serem escassos no que se refere à determinação de pontos de corte para o HOMA2, provavelmente por ter sido publicado mais recentemente para o HOMAl, o número de publicações ainda é crescente em diversos países, devendo-se atentar para os fatores de influência na SI, de modo a garantir maior adequação do ponto de corte a ser utilizado.

\section{REFERÊNCIAS}

1. Matthews DR. Insulin resistance and $\beta$-cell function - a clinical perspective. Diabetes Obes Metab. 2001;3:28-33.

2. Porte DJR, Kahn SE. Cell dysfunction and failure in type 2 diabetes potential mechanisms. Diabetes. 2001;50:160-3.

3. Ye J. Role of insulin in the pathogenesis of free fatty acid-induced insulin resistance in skeletal muscle. Endocr Metab Immune Disord Drug Targets. 2007;7:65-74.

4. Mlinar B, Marc J, Janez A, Pfeifer M. Molecular mechanisms of insulin resistance and associated diseases. Clin Chim Acta. 2007;375:20-35.

5. Facchini FS, Hua N, Abbasi F, Reaven GM. Insulin resistance as a predictor of age-related diseases. J Clin Endocrinol Metab. 2001;86:3574-8.

6. Weyer C, Tataranni PA, Bogardus C, Pratley RE. Insulin resis tance and insulin secretory dysfunction are independent predictors of worsening of glucose tolerance during each stage of type 2 diabetes development. Diabetes Care. 2001;24:89-94.

7. Resnick HE, Jones K, Ruotolo G, Jain AK, Henderson J, Lu W, et al. Insulin Resistance, the metabolic syndrome, and risk of incident cardiovascular disease in nondiabetic american indians. Diabetes Care. 2003;26:861-7.

8. Kim JA, Montagnani M, Koh KK, Quon MJ. Reciprocal relationships between insulin resistance and endothelial dysfunction: molecular and pathophysiological mechanisms. Circulation. 2006;18:1888-904.

9. Bigazzi R, Bianchi S. Insulin resistance, metabolic syndrome and endothelial dysfunction. J Nephrol. 2007;20:10-4. 
10. Golden SH, Folsom AR, Coresh J, Sharrett AR, Szklo M, Brancati F. Risk factor groupings related to insulin resistance and their synergistic effects on subclinical atherosclerosis: The Atherosclerosis Risk in Communities Study. Diabetes. 2002;51:3069-76.

11. Bonora E, Formentini G, Calcaterra F, Lombardi S, Marini F, Zenari $L$, et al. HOMA-estimated insulin resistance is an independent predictor of cardiovascular disease in type 2 diabetic subjects: prospective data from the Verona Diabetes Complications Study. Diabetes Care. 2002;25:1135-41.

12. Pirgon O, Kivrak AS. Evidence for association between insulin resistance and premature carotid atherosclerosis in childhood obesity. Pediatr Res. 2007;61:345-9.

13. Marques-Vidal P, Mazoyer E, Bongard V, Gourdy P, Ruidavets $J B$, Drouet $L$, et al. Prevalence of insulin resistance syndrome in southwestern France and its relationship with inflammatory and hemostatic markers. Diabetes Care. 2002;25:1371-7.

14. Pereira LO, Francischi RP, Lancha Jr AH. Obesidade: hábitos nutricionais, sedentarismo e resistência à insulina. Arq Bras Endocrinol Metab. 2003;47:111-27.

15. Rojo-Martinez G, Esteva I, de Adana SR, Catala M, Merelo MJ, Tinahones $\mathrm{F}$, et al. Patterns of insulin resistance in the general population of southeast Spain. Diabetes Res Clin Pract. 2004; 65:247-56.

16. Hermans MP, Pepersack TM, Godeaux LH, Beyer I, Turc AP. Prevalence and determinants of impaired glucose metabolism in frail elderly patients: The Belgian Elderly Diabetes Survey (BEDS). J Gerontol Med Sci. 2005;60:241-7.

17. Silva RC, Miranda WL, Chacra AR, Dib SA. Metabolic syndrome and insulin resistance in normal glucose tolerant brazilian adolescents with family history of type 2 diabetes. Diabetes Care. 2005;28:716-8

18. Lee JM, Okumura MJ, Davis MM, Herman WH, Gurney JG. Prevalence and determinants of insulin resistance among U.S. adolescents: a population-based study. Diabetes Care. 2006; 29:2427-32.

19. Bergman RN. Toward physiological understanding of glucose tolerance. Minimal-model approach. Diabetes. 1989;38:1512-27.

20. DeFronzo RA, Tobin JD, Andres R. Glucose clamp technique: a method for quantifying insulin secretion and resistance. Am J Physiol Endocrinol Metab. 1979;237:214-23.

21. Geloneze B, Tambascia MA. Avaliação laboratorial e diagnóstico da resistência insulínica. Arq Bras End Metab. 2006;50:208-15.

22. Matthews DR, Hosker JP, Rudenski AS, Naylor BA, Treacher DF, Turner RC. Homeostasis model assessment: insulin resistance and B-cell function from fasting plasma glucose and insulin concentrations in man. Diabetologia. 1985;28:412-9.

23. Haffner SM, D'Agostino R, Saad MF, Rewers M, Mykkanen L, Selby $J$, et al. Increased insulin resistance and insulin secretion in nondiabetic african-americans and hispanics compared with non-hispanic whites. The Insulin Resistance Atherosclerosis Study. Diabetes. 1996;45:742-8.

24. Lee S, Choi S, Kim HJ, Chung YS, Lee KW, Lee HC, et al. Cuto$\mathrm{ff}$ values of surrogate measures of insulin resistance for metabolic syndrome in korean non-diabetic adults. J Korean Med Sci. 2006;21:695-700.

25. Bonora E, Kiechl S, Willeit J, Oberhollenzer F, Egger G, Meigs $\mathrm{JB}$, et al. Insulin resistance as estimated by homeostasis model assessment predicts incident symptomatic cardiovascular disease in caucasian subjects from the general population: The Bruneck Study. Diabetes Care. 2007;30:318-24.

26. Turner RC, Holman RR, Hockaday TDR. Beta cell deficiency in maturity onset diabetes. Lancet. 1976;12:1272-4.
27. Turner R, Holman RR, Matthews D, Hockaday TR, Peto J. Insulin deficiency and insulin resistance interaction in diabetes: estimation of their relative contribution by feedback analysis from basal plasma insulin and glucose concentrations. Metabolism. 1979;28:1086-96.

28. Mathews DR, Holman RR, Peto J. Relative contributions of insulin deficiency and insulin resistance in maturity-onset diabetes. Lancet. 1982;13:596-8.

29. Wallace TM, Levy JC, Matthews DR. Use and abuse of HOMA modeling. Diabetes Care. 2004;27:1487-95.

30. Levy JC, Matthews DR, Hermans MP. Correct homeostasis model assessment (HOMA) evaluation uses the computer program. Diabetes Care. 1998;21:2191-2.

31. The Oxford Centre for Diabetes, Endocrinology \& Metabolism. Diabetes Trial Unit. HOMA Calculator. Disponível em: http:// www.dtu.ox.ac.uk/. Acesso em: 20/3/2007.

32. Bonora E, Targher G, Alberiche M, Bonadonna RC, Saggiani F, Zenere MB, et al. Homeostasis model assessment closely mirrors the glucose clamp technique in the assessment of insulin sensitivity: studies in subjects with various degrees of glucose tolerance and insulin sensitivity. Diabetes Care. 2000;23:57-63.

33. Vieira JGH. Avaliação dos potenciais problemas pré-analíticos e metodológicos em dosagens hormonais. Arq Bras Endocrinol Metab. 2002;46:9-15.

34. Kang ES, Yun YS, Park SW, Kim HJ, Ahn CW, Song YD, et al. Limitation of the validity of the homeostasis model assessment as an index of insulin resistance in Korea. Metabolism. 2005;54:206-11.

35. Wallace TM, Levy JC, Matthews DR. An increase in insulin sensitivity and basal beta-cell function in diabetic subjects treated with pioglitazone in a placebo-controlled randomized study. Diabetic Med. 2004;21:568-76.

36. Katsuki A, Sumida Y, Gabazza EC, Murashima S, Furuta M, Araki-Sasaki R, et al. Homeostasis model assessment is a reliable indicator of insulin resistance during follow-up of patients with type 2 diabetes. Diabetes Care. 2001;24:362-5.

37. Lansang MC, Williams GH, Carroll S. Correlation between the glucose clamp technique and the homeostasis model assessment in hypertension. Am J Hypertens. 2001;14:51-3.

38. Gungor N, Saad R, Janosky J, Arslanian S. Validation of surrogate estimates of insulin sensitivity and insulin secretion in children and adolescents. J Pediatr. 2004;144:47-55.

39. Vaccaro O, Masulli M, Cuomo V, Rivellese AA, Uusitupa M, Vessby $B$, et al. Comparative evaluation of simple indices of insulin resistance. Metabolism. 2004;53:1522-6.

40. Chang AM, Smith MJ, Bloem CJ, Galecki AT, Halter JB, Supiano MA. Limitation of the Homeostasis Model Assessment to predict insulin resistance and $\beta$-cell dysfunction in older people. J Clin Endocrinol Metab. 2006;91:629-34.

41. Albu JB, Kovera AJ, Allen L, Wainwright M, Berk E, Raja-Khan $\mathrm{N}$, et al. Independent association of insulin resistance with larger amounts of intermuscular adipose tissue and a greater acute insulin response to glucose in african american than in white nondiabetic women. Am J Clin Nutr. 2005;82:1210-7.

42. Roder ME. Reduced pancreatic $\beta$-cell compensation to the insulin resistance of aging: impact on proinsulin and insulin levels. J Clin Endocrinol Metab. 2000;85:2275-80.

43. Ryan AS, Nicklas BJ, Berman DM. Aerobic exercise is necessary to improve glucose utilization with moderate weight loss in women. Obesity. 2006;14:1064-72.

44. Donahue RP, Bean JA, Donahue RA, Goldberg RB, Prineas RJ. Insulin response in a triethnic population: effects of sex, ethnic origin, and body fat. Miami Community Health Study. Diabetes Care. 1997;20:670-6. 
45. Ryan AS, Nicklas BJ, Berman DM. Racial differences in insulin resistance and mid-thigh fat deposition in postmenopausal women. Obes Res. 2002;10:336-44.

46. McAuley K, Mann J. Thematic review series: patient-oriented research. Nutritional determinants of insulin resistance. J Lipid Res. 2006;47:1668-76.

47. McLaughlin TL, Reaven GM. Beyond type 2 diabetes: the need for a clinically useful way to identify insulin resistance. Am J Med. 2003;114:501-2.

48. Tresaco B, Bueno G, Pineda I, Moreno LA, Garagorri JM, Bueno M. Homeostatic Model Assessment (HOMA) index cut-off values to identify the metabolic syndrome in children. J Physiol Biochem. 2005;61:381-8.

49. Keskin M, Kurtoglu S, Kendirci M, Atabek ME, Yazici C. Homeostasis model assessment is more reliable than the fasting glucose/insulin ratio and quantitative insulin sensitivity check index for assessing insulin resistance among obese children and adolescents. Pediatrics. 2005;115:500-3.

50. Yeni-Komshian H, Carantoni M, Abbasi F, Reaven GM. Relationship between several surrogate estimates of insulin resistance and quantification of insulin-mediated glucose disposal in 490 healthy nondiabetic volunteers. Diabetes Care. 2000; 23:171-5.

51. Acosta B, Escalona MO, Maiz AG, Pollak FC, Leighton FP. Determinación del índice de resistencia insulínica mediante HOMA en una población de la región metropolitana de Chile. Rev Med Chile. 2002;130:1227-31.
52. Pozzan R, Pozzan R, Brandão AA, Magalhães MEC, Brandão AP. Níveis de insulina e HOMA em uma amostra da cidade do Rio de Janeiro. Estudo do Rio de Janeiro. Rev Socerj. 2003;16:75-5.

53. Ghiringhello MT, Vieira JG, Tachibana TT, Ferrer C, Maciel RMB, Oliveira CHRM, et al. Distribution of HOMA-IR in brazilian subjects with different body mass indexes. Arq Bras Endocrinol Metab. 2006;50:573-4.

54. Bonora E, KiechI S, Willeit J, Oberhollenzer F, Egger G, Targher $\mathrm{G}$, et al. Prevalence of insulin resistance in metabolic disorders: the Bruneck Study. Diabetes. 1998;47:1643-9.

55. Ascaso JF, Romero P, Real JT, Priego A, Valdecabres C, Carmena R. Cuantificación de insulinoresistencia con los valores de insulina basal e índice HOMA en una población no diabética. Med Clin. 2001;117:530-3.

56. Geloneze B, Repetto EM, Geloneze SR, Tambascia MA, Ermetice MN. The threshold value for insulin resistance (HOMAIR) in an admixtured population. IR in the Brazilian Metabolic Syndrome Study. Diabetes Res Clin Prac. 2006;72:219-20.

\section{Endereço para correspondência:}

Ana Carolina Junqueira Vasques

Av. PH Rolfs, s/n - Departamento de Nutrição e Saúde

36571-000 Viçosa, MG

E-mail: anacarolinavasques@yahoo.com.br 